\title{
Challenges in the Current Management of Hepatocellular Carcinoma
}

\author{
Soumya Jogi ${ }^{1}$, Radha Varanasi ${ }^{2}$, Sravani S Bantu ${ }^{3}$, Sudha Saduvala ${ }^{4}$, Ashish Manne ${ }^{* 5}$ \\ ${ }^{1}$ Department of Internal Medicine, Alluri Sitarama Raju Academy of Medical Sciences, Eluru, 534005, India \\ ${ }^{2}$ Department of Internal Medicine, St. Vincent's Birmingham, Birmingham, 35235, USA \\ ${ }^{3}$ Department of Internal Medicine, Baptist Medical Center, Montgomery, 36116, USA \\ ${ }^{4}$ Department of Internal Medicine, Dameron Hospital, Stockton, 95203, USA \\ ${ }^{5}$ Department of Medical Oncology, The James Comprehensive Cancer Center, Ohio State University, 43210, USA \\ *Corresponding author: Ashish Manne; ashish.manne@osumc.edu
}

Received 11 March 2021;

Accepted 28 March 2021;

Published 10 April 2021

\begin{abstract}
Management of hepatocellular carcinoma (HCC) is complicated. Barcelona Clinic Liver Cancer (BCLC) staging system is widely used in risk stratifying HCC. It is different from anatomic staging (TNM) used in other cancers and is based on the liver function (Child-Pugh Score) and performance status at diagnosis along with tumor characteristics like size/number of primary, vascular invasion, and distant metastasis. Guidelines proposed by various liver societies help the treating physician select first-line therapy, but there are many limitations to them. Lack of reliable biomarkers that give objective information to monitor the response other than alpha-fetoprotein or radiological response is hurting the management strategies. There are no ideal predictors for recurrence and residual microscopic disease, especially after locoregional therapy (LRT) like surgical resection, ablation, transarterial chemoembolization (TACE), transarterial radioembolization (TARE), and stereotactic radiation therapy (SBRT). Also, there is no convincing evidence to use adjunct therapy along with LRT in localized HCC. There is a need to identify the subset of HCC that would benefit from peri-procedural therapy. Recommendations for treating advanced HCC with macrovascular invasion is not uniform across the guidelines. Some propose LRT (TACE and/or TARE) or recommend systemic therapy only like tyrosinekinase inhibitors (TKI) or Immune-checkpoint inhibitors (ICI). A considerable portion of patients have poor liver function (Child-Pugh Score C) at diagnosis. In this era of medicine, we should give them options other than supportive care, but unfortunately, it is the preferred option. This population needs special attention in trials. In current practice, there only 2-3 classes of drugs available like TKI, ICI, and vascular endothelial growth factor (VEGF) inhibitors. There is a need to explore more classes of liver-friendly drugs in treating HCC, and the enrolment of patients in clinical trials must be advised in the guidelines.
\end{abstract}

Keywords: hepatocellular carcinoma, biomarkers, portal vein tumor thrombosis, adjunct therapy, immune-checkpoint inhibitors

\section{Background}

Medical management of HCC is a daunting task, especially in the advanced or intermediate stages. In clinical practice, multidisciplinary tumor board consensus heavily influenced by locally available treatment options (like ablation, TACE, TARE, SBRT, and surgery) is followed rather than any specific guidelines. With very few options approved and no-good biomarkers to depend on, we are not able to get desirable results in managing these patients. It is high time we invest in designing effective management of complicated HCC patients with portal vein tumor thrombosis, poor liver function, and post-procedural therapy. There is a need to expand the class of drugs and non-invasive biomarkers in HCC.

\section{Introduction}

Hepatocellular carcinoma (HCC) is one of the leading causes of cancer-related deaths worldwide, and its incidence is rising in recent years ${ }^{[1,2]}$. Liver disease study groups around the world have proposed guidelines to manage HCC ${ }^{[3-8]}$. They differ not only in staging the HCC but also in the preferred treatment options. Surgical resection, transarterial chemoembolization (TACE), ablation (radiofrequency or microwave), transarterial radioembolization (TARE), stereotactic body radiation therapy (SBRT), and external beam radiation therapy (EBRT) represent locoregional therapy (LRT). Systemic therapy is proposed in advanced stage HCC like macrovascular invasion (MVI) and distant metastasis. In early-stage tumors, they might be used in patients with poor liver function based on Child-Pugh Score (CP) or performance status (PS). 


\section{Aim of the review}

The majority of the patients who present to medical oncologists are unresectable cancers ranging from intermediate to terminal stages with limited options available to make a meaningful difference. In this review, we shed light on the critical areas which need immediate attention in managing HCC, including lack of reliable biomarkers (for risk stratification and monitoring the treatment), effective adjuvant therapy strategies, inadequate systemic options, tumors with macrovascular invasion, and patients with compromised liver function.

\section{Methods}

This systematic review was performed in January 2021using available databases: PubMed, Medline, Cochrane Library, Embase, and ClinicalTrials.gov. Unpublished data presented at international congresses as abstracts (American Society of Clinical Oncology (ASCO) and European Society of Medical Oncology (ESMO)) were also searched. The keywords used for the search were "Hepatocellular carcinoma" AND "management" OR "systemic therapy" OR "biomarkers" OR "Child-Pugh C" OR "clinical trials" OR "macrovascular invasion."

\section{Discussion}

\section{Biomarkers in Clinical Practice}

None of the guidelines proposed so far stressed using any biomarker for risk assessment or treatment recommendation. There are no prognostic markers in the current clinical practice to predict worse outcomes and monitor the response to treatment for HCC. Imaging (CT scan or MRI) and serum alfa-fetoprotein (AFP) levels are often used in monitoring the response to treatment are not always dependable. Diagnosis by imaging in the majority of cases removes the luxury of using traditional immunohistochemistry (IHC), or next-generation sequencing used to identify useful biomarkers like Her2 in breast cancer and MMR in colon cancer. As detailed in the table 1, biomarkers can be serum/blood-based testing ${ }^{[9-17]}$, genetic data (IHC or sequencing, ${ }^{[16-23]}$, mutations in circulating tumor cells (CTC) ${ }^{[24]}$, or treatment specific (sorafenib ${ }^{[25,26]}$, TARE ${ }^{[27]}$ or SBRT ${ }^{[28]}$ ). Most of these markers did not reach the clinical practice as of today.

Table 1: Biomarkers in HCC

\begin{tabular}{|c|c|}
\hline Serum based & $\begin{array}{l}\text { Platelet count (low) } \\
\text { Mean plasma volume (high) } \\
\text { HIV (seropositive) } \\
\text { AFP/AFP -L3 } \\
\text { Osteopontin } \\
\text { Des-gamma-carboxy prothrombin (DCP) } \\
\text { Glutamine synthetase } \\
\text { Gamma globulins (in CPA with Cirrhosis) } \\
\text { UL-16 binding protein } 1 \text { (ULBP1) in exosomes and as a free protein }(>2000 \mathrm{pg} / \mathrm{ml} \text { ) } \\
\text { Anti-p16a, anti-CD25a and anti-FOXP3 IgG } \\
\text { Exosomes - miR -122, miR-21 }\end{array}$ \\
\hline $\begin{array}{l}\text { Tissue based testing. } \\
\text { (IHC or sequencing) }\end{array}$ & $\begin{array}{l}\text { Transketolase (TKT) } \\
\text { Olfactomedin } 2 \text { (OLFM2) } \\
\text { Acyl-CoA dehydrogenase (ACAD) } \\
\text { Carnitine palmitoyltransferase } 2 \text { (CPT2) } \\
\text { Adhesion G Protein-Coupled Receptor B1 (ADGRB1) } \\
\text { Twist Family BHLH Transcription Factor } 1 \text { (TWIST1) } \\
\text { Glutamine synthetase } \\
\text { Glypican } 3 \\
\text { General stress protein, } \\
\text { Enhancer of zeste homologue } 2 \text { (EZH2) } \\
\text { Heat shock protein } 70 \text { (HSP 70) }\end{array}$ \\
\hline Mutations on CTC & $\begin{array}{l}\text { TP53 } \\
\text { Cyclin-dependent kinase inhibitor 2A (CDK2NA) } \\
\text { Telomerase Reverse Transcriptase (TERT), } \\
\text { Catenin beta } 1 \text { (CTNB1) } \\
\text { AXIN1 AT-rich interaction domain 1A (ARID1A) } \\
\text { Apolipoprotein B (APOB) } \\
\text { Splicing factor 3b subunit } 1 \text { (SF3B1) }\end{array}$ \\
\hline Sorafenib related & $\begin{array}{l}\text { Inter Leukin-17A } \\
\text { Hepatocyte growth factor [HGF] } \\
\text { Fibroblast growth factor [FGF] } \\
\text { Vascular endothelial growth factor receptor-1 } \\
\text { CD117 } \\
\text { Angiopoietin-2 }\end{array}$ \\
\hline TARE related & $\begin{array}{l}\text { Image response } \\
\text { Segmental treatment }\end{array}$ \\
\hline SBRT related & $\begin{array}{l}\text { Child-Pugh score } \\
\text { Portal vein tumor thrombosis }\end{array}$ \\
\hline
\end{tabular}




\section{Tumor size}

Tumor response

Tissue-based biomarkers can be identified by IHC (phenotypic), while the expression of the genes (genotypic) can be identified by sequencing or FISH. Identification of specific mutations in circulating tumor cells (CTC) technology can be used in risk stratifying at the diagnosis but also to monitor the treatment by following the mutational burden serially. Histopathology of HCC is often ignored, and its impact on outcomes is underrated. The latest classification of it into four types, steatohepatitic, clear cell, fibrolamellar, and scirrhous was proposed ${ }^{[29]}$. Combining this classification with biomarkers can aid a clinically significant classification of HCC. There is a need to study the effectiveness of each LRT on specific histology type.

\section{Adjunct therapy}

According to the Surveillance, Epidemiology, and End Results (SEER) database, the five-year survival rate of localized HCC (along with Intrahepatic Bile Duct Cancer) is a mere 34\%, which is surprisingly worse than localized pancreatic cancer $(39 \%)$. The prevalence of bile duct cancer is very low. It is safe to assume that the recurrence rate and survival rate of localized HCC are not as good as colon cancer or breast cancer, where adjuvant therapy is standard in the majority of the cases. In another study, the 10-year survival rate was only $10 \%$ in patients who received just LRT, which is alarming ${ }^{[30]}$.

Unlike breast or colon cancer, the available guidelines do not have enough pre or post-procedural options for the patients that receive LRT in HCC. We need to intensify the debate on adjuvant or neoadjuvant therapy in HCC. The first step in that direction is to identify the concerning risk factors for treatment failure and recurrence. A comprehensive system that utilizes the biomarkers (as mentioned above) along with traditional risk factors (of HCC) like liver function and pathological factors is needed for it. Even though there is some evidence of adding systemic therapy to TACE, most of the guidelines do not encourage it ${ }^{[31]}$. Autologous cytokine-induced killer (CIK) is used after LRT like surgery in some Asian countries, but it has not been studied in Europe or North America ${ }^{[32]}$. Table 2 gives a list of some of the adjuvant therapy trials after LRT, like resection and ablation, and if these trials are positive, it will open a door for us. This further emphasizes encouraging physicians to start clinical trials or enroll the patients in the available trials.

Table 3: Current trials in HCC with adjunct therapy

\begin{tabular}{|c|c|c|c|c|c|}
\hline LRT & $\begin{array}{l}\text { ClinicalTrials. } \\
\text { gov Identifier }\end{array}$ & Interventional arm & Comparative arm & Phase & Primary outcome \\
\hline Ablation & NCT04178642 & Idarubicin-Lipiodol & None - Single arm study & II & $\begin{array}{l}\text { Survival without recurrence in } \\
1 \mathrm{yr}\end{array}$ \\
\hline \multirow[b]{4}{*}{ RFA/Resection } & NCT03383458 & Nivolumab & Placebo & III & PFS \\
\hline & NCT04102098 & $\begin{array}{l}\text { Atezolizumab }+ \\
\text { Bevacizumab }\end{array}$ & Active Surveillance & III & PFS \\
\hline & NCT03867084 & Pembrolizumab & Placebo & III & PFS \& OS \\
\hline & NCT03847428* & $\begin{array}{l}\text { Durvalumab and } \\
\text { Bevacizumab }\end{array}$ & $\begin{array}{l}\text { Durvalumab + placebo \& } \\
\text { Placebo alone }\end{array}$ & III & PFS \\
\hline \multirow{6}{*}{ TACE } & NCT03608878 & $\begin{array}{l}\text { Adagloxad } \\
\text { simolenin/OBI-821 }\end{array}$ & Active Surveillance & II & $\begin{array}{l}\text { PFS Time-to-intrahepatic total } \\
\text { tumor volume progression }\end{array}$ \\
\hline & NCT03592706 & $\begin{array}{l}\text { Autologous Immune } \\
\text { Killer Cells }\end{array}$ & Active Surveillance & II/III & Change in tumor size PFS \\
\hline & NCT04340193 & $\begin{array}{l}\text { Nivolumab + } \\
\text { Ipilimumab } \\
\text { (Arm A) }\end{array}$ & $\begin{array}{l}\text { Nivolumab + Ipilimumab } \\
\text { (placebo) (Arm B) \& Nivolumab } \\
\text { (placebo)+ Ipilimumab (Arm C) }\end{array}$ & III & $\begin{array}{l}\text { TTTP will be assessed by } \\
\text { BICR: Arm A versus Arm C } \\
\text { OS Arm A versus Arm C }\end{array}$ \\
\hline & NCT04268888 & Nivolumab & No systemic treatment & $\mathrm{II} / \mathrm{III}$ & $\begin{array}{l}\text { OS - phase III primary } \\
\text { outcome, TTTP - phase II } \\
\text { outcome }\end{array}$ \\
\hline & NCT04246177 & $\begin{array}{l}\text { Pembrolizumab }+ \\
\text { Lenvatinib }\end{array}$ & Placebo (oral \& IV) & III & PFS per RECIST 1.1 OS \\
\hline & NCT03778957 & $\begin{array}{l}\text { Durvalumab + } \\
\text { Bevacizumab } \\
(\text { Arm B) }\end{array}$ & $\begin{array}{l}\text { Durvalumab + Placebo (Arm A) } \\
\text { \& Placebo }(\text { Arm C) }\end{array}$ & III & PFS - Arm B vs Arm C \\
\hline \multirow[t]{3}{*}{ TARE } & NCT04541173 & $\begin{array}{l}\text { Atezolizumab + } \\
\text { bevacizumab }\end{array}$ & No systemic treatment & II & PFS per RECIST 1.1 \\
\hline & NCT03099564 & Pembrolizumab & None - Single arm study & I & PFS per RECIST 1.1 \\
\hline & NCT03033446 & Nivolumab & None - Single arm study & II & ORR \\
\hline \multirow[t]{5}{*}{ SBRT } & NCT03203304 & $\begin{array}{l}\text { Ipilimumab + } \\
\text { Nivolumab }\end{array}$ & Nivolumab & $\mathrm{I}$ & $\begin{array}{l}\text { Number of participants } \\
\text { with adverse events }\end{array}$ \\
\hline & NCT01730937 & Sorafenib & Soragenib alone (No SBRT) & III & OS \\
\hline & NCT03316872 & Pembrolizumab & None - Single arm study & II & ORR \\
\hline & NCT03482102 & $\begin{array}{l}\text { Tremelimumab + } \\
\text { Durvalumab }\end{array}$ & None - Single arm study & II & ORR \\
\hline & NCT02906397 & Galunisertib & None - Single arm Study & $\mathrm{I}$ & Safety \\
\hline
\end{tabular}




\begin{tabular}{|l|l|l|l|l|l|}
\hline & NCT04387695 & Sorafenib + TACE & $\begin{array}{l}\text { Only Sorafenib (no SBRT or } \\
\text { TACE) }\end{array}$ & III & PFS \\
\cline { 2 - 6 } & NCT01730937 & With Sorafenib & Sorafenib alone & III & OS \\
\hline Surgery & $\begin{array}{l}\text { NCT03916627 } \\
\text { (neoadjuvant) }\end{array}$ & Cemipilumab & None- Single arm study & II & $\begin{array}{l}\text { Significant tumor necrosis } \\
\text { (STN) at time of surgery }\end{array}$ \\
\hline Electroporation & $\begin{array}{l}\text { ClinicalTrials. } \\
\text { gov Identifier }\end{array}$ & Nivolumab & Single arm study & II & Local PFS \\
\hline
\end{tabular}

OS - Overall Survival; PFS - progression-free survival; RECIST 1.1 - Response Evaluation Criteria in Solid Tumors Version 1.1; TTTP - Time to TACE progression; BICR - Blinded independent central review; STN - Significant tumor necrosis

\section{Macrovascular invasion}

MVI in HCC refers to invasion portal vein or hepatic veins by the tumor. It is seen in $20 \%$ of the new HCC patients, and close to $50 \%$ of the unresectable HCC without it at presentation will eventually develop it ${ }^{[33]}$. MVI is associated with a grim prognosis as it impairs the blood flow to (and from) the liver; increases the risk of tumor spread; makes the tumor unresectable and ineligible to transplant; causes portal hypertension and profound liver failure [33]. For PVTT, the location of tumor invasion is important, the main trunk or its branches (first vs second order vs beyond) as the efficacy of LRT like TACE or TARE may depend on it.

Almost all the guidelines favor using TKI in HCC with MVI, as there is strong evidence for it except with Ramucirumab (by posthoc analysis of the trials) ${ }^{[33]}$. In an MDTB approach, the use of LRT, especially TARE, is not uncommon, and the success of such procedures is debatable. In TACE, the hepatic artery is blocked, which may cause liver failure and post-embolization syndrome in patients with blocked hepatic or portal veins. It is effective in selected cases (with branch vessel thrombus) ${ }^{[33,34]}$. Survival advantage with TARE was found only in patients with $\mathrm{CP}$ A and branch vessel disease ${ }^{[3,35]}$. SBRT seems to have a good response than TARE (with Y90), but that did not convert into any survival advantage ${ }^{[36]}$. Surgery, including hepatectomy with en bloc vascular resection or thrombectomy, may be effective in possible cases ${ }^{[37]}$. As in KLCG guidelines, where TACE and EBRT combination is proposed, we need to start assessing the use of adjuvant and neoadjuvant therapy in HCC with VMI. A combination of TARE with Sorafenib was studied in a number of studies but not specifically in patients with MVI but in unresectable tumors ${ }^{[35]}$. Just as with SBRT, the response rate is good, but it did not translate to a survival advantage.

It is not clear if there is any impact on the outcome or if the approach should be different for the hepatic vein invasion compared to the portal vein. The reluctance to start TKI (with multiple undesirable side effects) and temptation to use TARE or TACE in the clinical practice is comprehensible, especially in healthy patients, but the evidence is not favorable for such an approach. Pending reliable prospective trials, the success of ICI should prompt its use with LRT in non-metastatic HCC with MVI. The timing of such a combination can be concurrent or sequential.

\section{HCC with Child-Pugh C}

Management of patients with poor liver function (CP C) needs attention too. Such patients are categorized as terminal stage irrespective of their pathological features and PS. Major trials for both systemic and local therapies traditionally excluded such patients leaving us practically with no options for their management. If transplant-ineligible, supportive care is advised across the guidelines.

In our study accepted for ASCO this year (as discussed above), the use of ICI in terminal stage patients was the prime reason for non-adherence to the guidelines (which suggested BSC). When we further analyzed those patients, seven (7/13) patients were classified into that group due to $\mathrm{CP} \mathrm{C}$ only, while the rest were due to PS. Four out of those seven (4/7) CP C patients were treated with ICI ( 3 Nivolumab \& 1 Pembrolizumab). One had a partial response for close to 10 months while the other had stable disease for 5 months, and none of them experienced grade 3/4 adverse events ${ }^{[38]}$. There are two takeaways from this limited study, (a) close to $50 \%$ of patients had PR or SD for a good 45months; (b) ICI is tolerable in CP C patients. There are other studies in the literature which support the tolerability of ICI in terminal stages ${ }^{[39,40]}$. The evidence on the efficacy of ICI in patients with poor liver function is limited by the studies (both prospective and retrospective) done on them.

A strategy to support the patients in terminal disease with reasonable PS should include a multidisciplinary approach with hepatologists leading the way and interventional radiologist or endoscopist supporting the oncologist. In patients with low tumor burden and treatable causes like HBV or HBC, addressing the etiology may give more time for disease control. Managing complications of liver failure like ascites (with diuretics), hepatic encephalopathy (with lactulose), and variceal hemorrhage (with endoscopic management), duct obstruction by the tumor (with $\mathrm{ERCP} /$ stenting or external drains) may give a fighting chance to save the patients. Trials must start including this subset of patients. Such trials might help manage the patients who start with good liver function but worsens later secondary to increasing tumor burden or other non-tumor-related causes (like cirrhosis).

\section{Systemic therapy}

Systemic therapy was also a weak link in the management of HCC until Sorafenib got approved in 2008. In recent years there has been considerable progress in this front, and we have 2-3 classes of drugs available now - tyrosine kinase inhibitor (TKI), vascular endothelial growth factor (VEGF) inhibitors, and immunecheckpoint inhibitor (ICI). Current options for TKIs are Sorafenib, Lenvatinib, Regorafenib, and Cabozantinib ${ }^{[41-44]}$. Until recently, the only VEGF inhibitor used was Ramucirumab (in AFP > $400)^{[45]}$.

Convincing evidence for ICI in the first line was given by IMbrave150 trial, where the combination of Atezolizumab and Bevacizumab did well against Sorafenib (Overall survival at 12 months was $67.2 \%$ vs $54.6 \%)^{[46]}$. In the Checkmate 459 trial, when compared to Sorafenib, nivolumab had a better objective response rate (ORR), more complete responses (CR), and fewer severe adverse events, but it did not reach the pre-determined median overall survival threshold. In the first trial on nivolumab for HCC. In Checkmate 40 (an open-label, non-comparative, phase 1/2 dose escalation and expansion trial), Sorafenib treated patients were added in the expansion phase. Durable ORR was seen in both sets of patients and was tolerable and better in the Sorafenib treated grou ${ }^{[47]}$. It was approved for the first line in patients who may not tolerate Sorafenib. On the other hand, Pembrolizumab was tested in second-line testing after Sorafenib and was compared with best 
supportive care (in keynote 240$)^{[48]}$. The trial did not reach prespecified targets but had good ORR.

It is disheartening to see limited systemic options in this deadly disease. There are many ongoing trials involving ICI or its combination with TKI (like HIMALAYA, LEAP-002, COSMIC312, and CHECKMATE 9DW). In the earlier versions of guidelines, enrolling patients in clinical trials was encouraged but not in any latest guidelines. There is a need to encourage clinicians to enroll patients in first or second-line trials before a significant decline in their PS or liver function.

\section{Conclusions}

The approach to HCC with MVI and CP C needs attention as the available options (in the guidelines) are not encouraging. There is a need to design and enroll patients actively in trials involving adjunct therapies, especially in early/intermediate stage HCC. Biomarkers other than traditional AFP should be used in early diagnosis, predict recurrence and monitor therapy are needed for effective management of HCC.

\section{List of abbreviations}

Hepatocellular carcinoma (HCC)

Locoregional therapy (LRT)

Transarterial chemoembolization (TACE)

Transarterial radioembolization (TARE)

Stereotactic radiation therapy (SBRT)

Immune-checkpoint inhibitors (ICI)

Tyrosine-kinase inhibitors (TKI)

Macrovascular invasion (MVI)

Child Pugh Score (CP)

\section{Conflicts of Interest}

The author(s) declare(s) that there is no conflict of interest regarding the publication of this paper.

\section{Funding Statement}

No funding was used for this project

\section{Authors' contributions}

Authors SJ, RV, SB and SS did the literature search and contributed to manuscript writing. AM supervised and reviewed the manuscript. All authors read and approved the final manuscript.

\section{References}

[1] Jemal A, Ward EM, Johnson CJ, et al. Annual Report to the Nation on the Status of Cancer, 1975-2014, Featuring Survival. J Natl Cancer Inst. 2017;109(9).

[2] Singal AG, Lampertico P, Nahon P. Epidemiology and surveillance for hepatocellular carcinoma: New trends. $J$ Hepatol. 2020;72(2):250-261.

[3] Marrero JA, Kulik LM, Sirlin CB, et al. Diagnosis, Staging, and Management of Hepatocellular Carcinoma: 2018 Practice Guidance by the American Association for the Study of Liver Diseases. Hepatology. 2018;68(2):723-750.

[4] EASL Clinical Practice Guidelines: Management of hepatocellular carcinoma. J Hepatol. 2018;69(1):182236.
[5] Kokudo N, Takemura N, Hasegawa K, et al. Clinical practice guidelines for hepatocellular carcinoma: The Japan Society of Hepatology 2017 (4th JSH-HCC guidelines) 2019 update. Hepatol Res. 2019;49(10):11091113.

[6] Omata M, Cheng AL, Kokudo N, et al. Asia-Pacific clinical practice guidelines on the management of hepatocellular carcinoma: a 2017 update. Hepatol Int. 2017;11(4):317-370.

[7] 2018 Korean Liver Cancer Association-National Cancer Center Korea Practice Guidelines for the Management of Hepatocellular Carcinoma. In: Gut Liver. Vol 13.2019:227-299.

[8] NCCN clinical practice guidelines in oncology.

[9] Scheiner B, Kirstein M, Popp S, et al. Association of Platelet Count and Mean Platelet Volume with Overall Survival in Patients with Cirrhosis and Unresectable Hepatocellular Carcinoma. Liver Cancer. 2019;8(3):203217.

[10] Easom NJW, Marks M, Jobe D, et al. ULBP1 Is Elevated in Human Hepatocellular Carcinoma and Predicts Outcome. Front Oncol. 2020;10:971.

[11] Wang J, Xu Y, Wang Y, Zhang X, Zhang G. Further Study of Circulating Antibodies to P16, CD25 and FOXP3 in Hepatocellular Carcinoma. Onco Targets Ther. 2019;12:10487-10493.

[12] Franck M, Schutte K, Malfertheiner P, Link A. Prognostic value of serum microRNA-122 in hepatocellular carcinoma is dependent on coexisting clinical and laboratory factors. World $J$ Gastroenterol. 2020;26(1):86-96.

[13] Pinato DJ, Allara E, Chen TY, et al. Influence of HIV Infection on the Natural History of Hepatocellular Carcinoma: Results From a Global Multicohort Study. J Clin Oncol. 2019;37(4):296-304.

[14] Yoo JJ, Chung GE, Lee JH, et al. Sub-classification of Advanced-Stage Hepatocellular Carcinoma: A Cohort Study Including 612 Patients Treated with Sorafenib. In: Cancer Res Treat. Vol 50.2018:366-373.

[15] Cacciola I, Filomia R, Alibrandi A, et al. Hypergammaglobulinemia is a strong predictor of disease progression, hepatocellular carcinoma, and death in patients with compensated cirrhosis. Liver Int. 2018;38(7):1220-1229.

[16] Adebayo Michael AO, Ko S, Tao J, et al. Inhibiting Glutamine-Dependent mTORC1 Activation Ameliorates Liver Cancers Driven by beta-Catenin Mutations. Cell Metab. 2019;29(5):1135-1150 e1136.

[17] Liu P, Lu D, Al-Ameri A, et al. Glutamine synthetase promotes tumor invasion in hepatocellular carcinoma through mediating epithelial-mesenchymal transition. Hepatol Res. 2020;50(2):246-257.

[18] Piñero F, Dirchwolf M, Pessôa MG. Biomarkers in Hepatocellular Carcinoma: Diagnosis, Prognosis and Treatment Response Assessment. In: Cells. Vol 9.2020.

[19] Zhang R, Ye J, Huang H, Du X. Mining featured biomarkers associated with vascular invasion in HCC by bioinformatics analysis with TCGA RNA sequencing data. Biomed Pharmacother. 2019;118:109274.

[20] Chen D, Feng X, Lv Z, et al. ACADS acts as a potential methylation biomarker associated with the proliferation and metastasis of hepatocellular carcinomas. Aging (Albany NY). 2019;11(20):8825-8844. 
[21] Duan J, Wu Y, Liu J, et al. Genetic Biomarkers For Hepatocellular Carcinoma In The Era Of Precision Medicine. In: J Hepatocell Carcinoma. Vol 6.2019:151166.

[22] Sato K, Meng F, Glaser S, Alpini G. Exosomes in liver pathology. J Hepatol. 2016;65(1):213-221.

[23] Chen LT, Martinelli E, Cheng AL, et al. Pan-Asian adapted ESMO Clinical Practice Guidelines for the management of patients with intermediate and advanced/relapsed hepatocellular carcinoma: a TOSESMO initiative endorsed by CSCO, ISMPO, JSMO, KSMO, MOS and SSO. Ann Oncol. 2020;31(3):334-351.

[24] Rice A, Del Rio Hernandez A. The Mutational Landscape of Pancreatic and Liver Cancers, as Represented by Circulating Tumor DNA. Front Oncol. 2019;9:952.

[25] Cho HJ, Kim SS, Nam JS, et al. Higher serum interleukin-17A levels as a potential biomarker for predicting early disease progression in patients with hepatitis B virus-associated advanced hepatocellular carcinoma treated with sorafenib. Cytokine. 2017;95:118125 .

[26] Kim HY, Lee DH, Lee JH, et al. Novel biomarker-based model for the prediction of sorafenib response and overall survival in advanced hepatocellular carcinoma: a prospective cohort study. BMC Cancer. 2018;18(1):307.

[27] Gordon AC, Gabr A, Riaz A, et al. Radioembolization Super Survivors: Extended Survival in Non-operative Hepatocellular Carcinoma. Cardiovasc Intervent Radiol. 2018;41(10):1557-1565.

[28] Que J, Kuo HT, Lin LC, et al. Clinical outcomes and prognostic factors of cyberknife stereotactic body radiation therapy for unresectable hepatocellular carcinoma. BMC Cancer. 2016;16:451.

[29] El Jabbour T, Lagana SM, Lee H. Update on hepatocellular carcinoma: Pathologists' review. World $J$ Gastroenterol. 2019;25(14):1653-1665.

[30] Sempokuya T, Wong LL. Ten-year survival and recurrence of hepatocellular cancer. Hepatoma Res. $2019 ; 5$.

[31] Huang Y, Chen B, Liu N, et al. Overall survival in response to sorafenib with transarterial chemoembolization for BCLC stage B hepatocellular carcinoma: propensity score analysis. Int $J$ Clin Pharmacol Ther. 2017;55(6):498-508.

[32] Xu L, Wang J, Kim Y, et al. A randomized controlled trial on patients with or without adjuvant autologous cytokine-induced killer cells after curative resection for hepatocellular carcinoma. In: Oncoimmunology. Vol 5.2016 .

[33] Qadan M, Kothary N, Sangro B, Palta M. The Treatment of Hepatocellular Carcinoma With Portal Vein Tumor Thrombosis. Am Soc Clin Oncol Educ Book. 2020;40:18.

[34] Luo J, Guo RP, Lai EC, et al. Transarterial chemoembolization for unresectable hepatocellular carcinoma with portal vein tumor thrombosis: a prospective comparative study. Ann Surg Oncol. 2011;18(2):413-420.

[35] Salem R, Lewandowski RJ, Mulcahy MF, et al. Radioembolization for hepatocellular carcinoma using
Yttrium-90 microspheres: a comprehensive report of long-term outcomes. Gastroenterology. 2010;138(1):5264.

[36] Rim CH, Kim CY, Yang DS, Yoon WS. Comparison of radiation therapy modalities for hepatocellular carcinoma with portal vein thrombosis: A meta-analysis and systematic review. Radiother Oncol. 2018;129(1):112122.

[37] Chok KS, Cheung TT, Chan SC, Poon RT, Fan ST, Lo CM. Surgical outcomes in hepatocellular carcinoma patients with portal vein tumor thrombosis. World $J$ Surg. 2014;38(2):490-496.

[38] Manne A MM, Escobar DE, et al. Compliance to the American Association for the Study of Liver Diseases (AASLD) guidelines and its impact on overall survival in patients with hepatocellular carcinoma. 2020.

[39] Rao N, Rizk EM, Hwang R, et al. Nivolumab for hepatocellular carcinoma (HCC) in a real-world context. J Clin Oncol. 2019;37(15).

[40] Scheiner B, Kirstein MM, Hucke F, et al. Programmed cell death protein-1 (PD-1)-targeted immunotherapy in advanced hepatocellular carcinoma: efficacy and safety data from an international multicentre real-world cohort. Aliment Pharmacol Ther. 2019;49(10):1323-1333.

[41] Llovet JM, Ricci S, Mazzaferro V, et al. Sorafenib in advanced hepatocellular carcinoma. The New England journal of medicine. 2008;359(4):378-390.

[42] Kudo M, Finn RS, Qin S, et al. Lenvatinib versus sorafenib in first-line treatment of patients with unresectable hepatocellular carcinoma: a randomised phase 3 non-inferiority trial. Lancet. 2018;391(10126):1163-1173.

[43] Bruix J, Qin S, Merle P, et al. Regorafenib for patients with hepatocellular carcinoma who progressed on sorafenib treatment (RESORCE): a randomised, doubleblind, placebo-controlled, phase 3 trial. Lancet. 2017;389(10064):56-66.

[44] Abou-Alfa GK, Meyer T, Cheng AL, et al. Cabozantinib in Patients with Advanced and Progressing Hepatocellular Carcinoma. The New England journal of medicine. 2018;379(1):54-63.

[45] Zhu AX, Kang YK, Yen CJ, et al. Ramucirumab after sorafenib in patients with advanced hepatocellular carcinoma and increased $\alpha$-fetoprotein concentrations (REACH-2): a randomised, double-blind, placebocontrolled, phase 3 trial. Lancet Oncol. 2019;20(2):282296.

[46] Finn RS, Qin S, Ikeda M, et al. Atezolizumab plus Bevacizumab in Unresectable Hepatocellular Carcinoma. The New England journal of medicine. 2020;382(20):1894-1905.

[47] El-Khoueiry AB, Sangro B, Yau T, et al. Nivolumab in patients with advanced hepatocellular carcinoma (CheckMate 040): an open-label, non-comparative, phase $1 / 2$ dose escalation and expansion trial. Lancet. 2017;389(10088):2492-2502.

[48] Finn RS, Ryoo BY, Merle P, et al. Pembrolizumab As Second-Line Therapy in Patients With Advanced Hepatocellular Carcinoma in KEYNOTE-240: A Randomized, Double-Blind, Phase III Trial. J Clin Oncol. 2020;38(3):193-202. 